USA. ${ }^{14}$ Ottawa Hospital Research Institute, Ottawa, Canada. ${ }^{15}$ Department of Clinical Epidemiology, Biostatistics and Bioinformatics, University of Amsterdam, Amsterdam, the Netherlands.

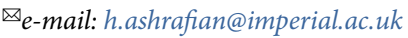

Published online: 8 June 2020

https://doi.org/10.1038/s41591-020-0941-1
References

1. Topol, E. J. Nat. Med. 25, 44-56 (2019)

2. US Food and Drug Administration. https://www.fda.gov/ medical-devices/digital-health/software-medical-device-samd (2018).

3. Bossuyt, P. M. et al. Br. Med. J. 351, h5527 (2015)

4. Bluemke, D. A. et al. Radiology https://doi.org/10.1148/ radiol.2019192515 (2019).

The EQUATOR Network. https://www.equator-network org/library/reporting-guidelines-under-development/

reporting-guidelines-under-development-for-other-study-designs /\#STARDAI (2019).
6. Liu, X. et al. Nat. Med. 25, 1467-1468 (2019).

7. Collins, G. S. \& Moons, K. G. M. Lancet 393, 1577-1579 (2019).

\section{Acknowledgements}

Infrastructure support was provided by the NIHR Imperial Biomedical Research Centre.

D.K., J.D.F. and A.K. are employees of Google Health. A.D. is an adviser at Google DeepMind/Health.

\title{
The QT interval in patients with COVID-19 treated with hydroxychloroquine and azithromycin
}

To the Editor - The SARS-CoV-2 pandemic has caused more than 1.6 million positive cases and more than 95,000 confirmed deaths as of 10 April $2020\left(\right.$ ref. $\left.^{1}\right)$. Although there are no approved drugs to prevent or treat SARS-CoV-2 infection ${ }^{2}$, a recent report suggested that the combination of hydroxychloroquine and azithromycin (HY/ AZ) may have a favorable effect on the clinical outcomes and viral loads of infected patients ${ }^{3}$; this resulted in massive adoption of the regimen by clinicians worldwide. However, both medications have been independently shown to increase the risk in other populations for QT-interval prolongation, drug-induced torsades de pointes (a form of polymorphic ventricular tachycardia) and drug-induced sudden cardiac death ${ }^{4-6}$. In our center, patients with the respiratory syndrome COVID-19 who are admitted for lower airway disease with features such as non-resolving cough, chest infiltrates on X-ray and persistent fever, with or without blood-oxygen desaturation, are treated with HY/AZ. We reviewed the charts and followed the corrected QT (QTc) interval in a consecutive cohort of 84 patients receiving the regimen. $\mathrm{HY}$ and $\mathrm{AZ}$ were administered orally for 5 days. HY was given at a dose of $400 \mathrm{mg}$ twice daily on the first day, followed by $200 \mathrm{mg}$ twice daily. AZ was given at a dose of $500 \mathrm{mg}$ per day. The average time of electrocardiograph (ECG) follow-up after HY/AZ exposure was $4.3 \pm 1.7$ days.

We observed prolongation of the QTc from a baseline average of $435 \pm 24 \mathrm{~ms}$ (mean \pm s.d.) to a maximal average value of $463 \pm 32 \mathrm{~ms}(P<0.001$ (one-sample $t$-test)), which occurred on day $3.6 \pm 1.6$ of therapy (Fig. 1). In a subset of nine (11\%) of those patients, the QTc was severely prolonged to $>500 \mathrm{~ms}$, a known marker of high risk of malignant arrhythmia and sudden cardiac death ${ }^{7}$. In this high-risk group, the QTc increased from a baseline average a

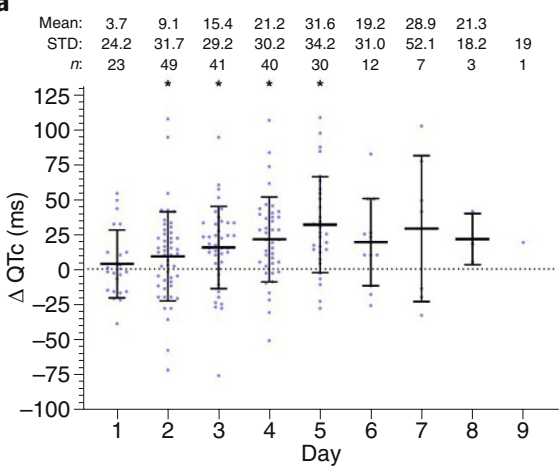

b

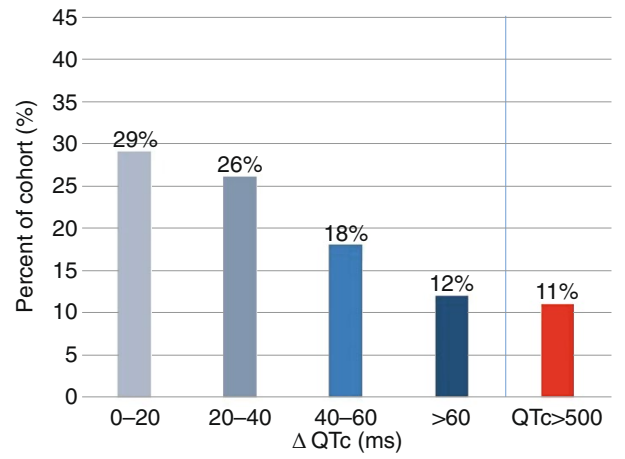

Fig. 1 | Changes in QTc on HY/AZ therapy. a, Change in QTc, presented as days after HY/AZ initiation. ${ }^{\star} P<0.01$, QTc compared with baseline QTc (one-sample $t$-test to compare each sample against a change in QTc ( $\Delta \mathrm{QTC}$ ) of 0 ms (i.e., no change from baseline), with adjustment for multiple testing). Each data point represents a single patient with a single ECG at any given interval (n). STD, standard deviation. b, Frequency of patients with various ranges of QTc prolongation (horizontal axis). Five cardiologists trained and experienced in QT measurement performed all ECG measurements. QT and RR measurements were validated by a senior cardiac electrophysiologist expert in QT measurements. QTc was corrected with the Bazett formula $\left(\mathrm{QTC}=\mathrm{QT} / \mathrm{RR}^{1 / 2}\right)$

of $447 \pm 30 \mathrm{~ms}$ to $527 \pm 17 \mathrm{~ms}(P<0.01$

(one-sample $t$-test)). There were no torsades de pointes events recorded for any patients, including those with a severely prolonged QTc. Four patients died from multi-organ failure, without evidence of arrhythmia and without severe QTc prolongation. 64 patients remained admitted and 16 patients were discharged. The clinical and epidemiological characteristics are presented in Supplementary Table 1.

The effectiveness of HZ/AZ in treating SARS-CoV-2 infection has been demonstrated in one small human study so far $^{2}$. Previously, the combination of $\mathrm{HY} / \mathrm{AZ}$ resulted in mild QTc prolongation when given to young healthy volunteers ${ }^{8}$. In our work, we found that in patients with COVID-19 who were treated with HY/AZ, the QTc was significantly prolonged. This discrepancy suggests that QT prolongation may be influenced by patient attributes such as the presence of co-morbidities and the severity of the disease $^{9}$. Of note, recent guidance suggested ECG screening with QTc assessment for patients with COVID-19 who are candidates for novel therapies, including $\mathrm{HY} / \mathrm{AZ}^{10}$. In our cohort, five of nine patients with severe QTc prolongation had a normal QTc at baseline. We therefore suggest that the QTc should be followed repeatedly in patients with COVID-19 who are treated with HY/AZ, particularly in those with co-morbidities and in those who are treated with other QT-prolonging medications.

\section{Ethics declaration}

The study was performed according to our Institutional Review Board guidance in 
accordance with the ethical standards laid down in the 1964 Declaration of Helsinki and its later amendments, with a waiver of informed consent for chart review.

\section{Data availability}

The data in this study will be shared upon request and approval will be designated by a data access committee. The data access committee comprises four authors and there is no restriction to data access.

\section{Editorial note: This article has been peer-reviewed.}

David S. Park, Larry A. Chinitz and Lior Jankelson $\bowtie$

Leon H. Charney Division of Cardiology, Cardiac Electrophysiology, NYU Langone Health, New York University School of Medicine, New York, NY, USA.

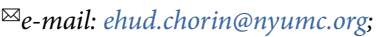

lior.jankelson@nyumc.org

Published online: 24 April 2020

https://doi.org/10.1038/s41591-020-0888-2

References

1. World Health Organization. https://www.who.int/emergencies/ diseases/novel-coronavirus-2019/situation-reports (2020).

2. Wang, M. et al. Cell Res. 30, 269-271 (2020).

3. Gautret, P. et al. Int. J. Antimicrob. Agents https://doi. org/10.1016/j.ijantimicag.2020.105949 (2020).

4. Morgan, N. D., Patel, S. V. \& Dvorkina, O. J. Clin. Rheumatol. 19 286-288 (2013).

5. HUANG, B. H. Pacing Clin. Electrophysiol. 30, 1579-1582 (2007)

6. Kezerashvili, A., Khattak, H., Barsky, A., Nazari, R. \& Fisher, J. D. J. Interv. Card. Electr. 18, 243-246 (2007).
7. Goldenberg, I. et al. Circulation 117, 2184-2191 (2008)

8. Hancox, J. C., Hasnain, M., Vieweg, W. V., Crouse, E. L. \& Baranchuk, A. Ther. Adv. Infect. Dis. 1, 155-165 (2013).

9. Fernandes, F. M., Silva, E. P., Martins, R. R. \& Oliveira, A. G. PLoS One 13, e0199028 (2018).

10. Giudicessi, J.R., Noseworthy, P.A., Friedman, P.A. \& Ackerman, M.J. Mayo Clin. Proc. https://doi.org/10.1016/j. mayocp.2020.03.024 (2020)

\section{Author contributions}

E.C. and L.J. contributed to the study design and data interpretation and writing of manuscript; M.D. contributed to statistical analysis; E.S., L.W. and R.B.-C. contributed to data-collection analysis; A.A., D.H., S.B., M.S, D.S.P. and L.A.C. contributed to critical revisions to the manuscript; and all authors reviewed and approved the final version of the manuscript.

Competing interests

The authors declare no competing interests. https://doi.org/10.1038/s41591-020-0888-2.

\section{Possible consequences of the COVID-19} pandemic on the use of biospecimens from

\section{cancer biobanks for research in academia and bioindustry}

To the Editor - The COVID-19 pandemic highlights the risks associated with the collection and processing of human biospecimens with an unknown status for the coronavirus SARS-CoV-2, whether for diagnostic, therapeutic or research purposes. Biosamples from patients with cancer, which continue to be collected and stored in biobanks during the pandemic, are likely to be infected with SARS-CoV-2. Apart from urine, all types of biospecimens (tissues, biofluids and swabs) and organs are potentially affected ${ }^{1-6}$. SARS-CoV-2 is likely to be inactivated in formalin-fixed, paraffin-embedded samples heated to $56^{\circ} \mathrm{C}\left(133^{\circ} \mathrm{F}\right)^{7}$. However, as SARS-CoV-2 survives on various types of surfaces, it is unclear whether this could also apply to cassettes containing formalin-fixed, paraffin-embedded samples ${ }^{8}$. For this reason, and because SARS-CoV-2 is highly infective, it is essential to prepare, store, handle and ship human samples to ensure that the people exposed to the biospecimens not only are familiar with the appropriate safety procedures for handling potentially infectious fluids or tissue samples but also are able and willing to implement them.

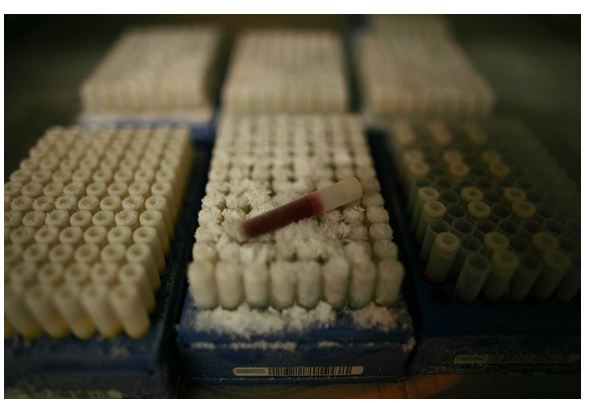

Image credit: Christopher Furlong/Getty Images News.

Universal precautions remain the best practice for the control of potential infection from human samples. Therefore, SARS-CoV-2-positive samples should not be marked accordingly, since these precautions apply to all biospecimens (as in the COVID-19 Biospecimen Guidelines of the University of California, San Francisco: https://research.ucsf.edu/covid19-biospecimen-guidelines). It is mandatory to work at biosafety level 2 (BSL-2) and to use class II biosafety workbenches (https:// www.cdc.gov/coronavirus/2019-ncov/lab/ lab-biosafety-guidelines.html). Reproductive work (e.g., viral culture, isolation or neutralization tests) should be carried out in laboratories with inward-directed airflow (BSL-3) (https://www.who.int/publicationsdetail/laboratory-biosafety-guidancerelated-to-coronavirus-disease-2019(covid-19)).

Many cancer biobanks, but also researchers, do not have access to the security facilities mentioned above. Introducing them would be costly, not only for biobanks but also for researchers from academia and biotech/biopharmaceutical companies requesting these samples and the associated clinical data. Under what conditions should biomaterials be collected from patients with cancer during and after the current COVID-19 pandemic? The relevant ethical and legal consequences of the tests must also be clarified. There is a need to specify which COVID-19-related symptoms, such as dry cough or fever, should be recorded, as well as who should record these data and until when. For avoidance of possible cross-contamination, an immediate and 